\title{
Leaf growth and stomatal sensitivity to ABA in droughted pepper plants
}

\begin{abstract}
The role of xylem sap abscisic acid (ABA) in regulating leaf growth and stomatal conductance was investigated on droughted pepper cultivars. Two pepper cultivars Capsicum annuum var. grossum, Bellboy and Capsicum annuum var. annuum 'Cili Padi' were established in growth chamber in soil compost mixture-filled polyvinyl-chloride tubes, 200 $\mathrm{mm}$ long and of an internal diameter of $105 \mathrm{~mm}$. Soil was fertilized and wet to field capacity during plant establishment. One group of plants was given one last watering and left unwatered for 9 days and another group of plants was watered daily. Under gradually decreasing soil moisture content, $C$. annuum var. grossum 'Bellboy' and C. annuum var. annuum 'Cili Padi' differed in their leaf water status, stomatal conductance, leaf growth and xylem sap ABA concentrations. The reduction in stomata and leaf growth was independent to the changes in the internal water relations as soil drying progressed. Stomatal conductance of 'Bellboy' began to decline within 2 days of cessation of watering, while 'Cili Padi' showed a reduction in this variable approximately 2 days later. Leaf growth of well watered 'Bellboy' was more rapid than that of 'Cili Padi'. When watering was stopped, 'Bellboy' showed an earlier inhibition of leaf growth. There were differences in the sensitivity of stomata and leaf growth to leaf and soil water status. Leaf growth was apparently responsive to variation in soil water availability in a range where leaf water status did not change. Both cultivars showed the same responses in stomatal conductance and leaf growth when expressed as a function of ABA concentration in the xylem. The study showed that under gradual water stress condition, any production of $\mathrm{ABA}$ from roots to be ineffective in triggering early stomatal closure and cessation of leaf growth.
\end{abstract}

Keyword: Drought; Capsicum; Stomatal conductance; Water relations; ABA 